\title{
Tendência da Temperatura e Precipitação na Península Antártica
}

\author{
Henderson Silva Wanderley ${ }^{1}$, Flávio Barbosa Justino², Gilberto Chuaco Sediyama ${ }^{2}$ \\ ${ }^{1}$ Departamento de Ciências Ambientais, Instituto de Floresta, \\ Universidade Federal Rural do Rio de Janeiro, Seropédica, RJ, Brasil. \\ ${ }^{2}$ Departamento de Engenharia Agrícola, Universidade Federal de Viçosa, Viçosa, MG, Brasil.
}

Recebido: 13/10/2013 - Aceito: 13/7/2015

\begin{abstract}
Resumo
A Península Antártica é uma das regiões que apresentou um acentuado aumento da temperatura do ar nas últimas décadas, sendo este maior que a média global. Deste modo, objetivou-se identificar tendências mensais em dados de temperatura extremas e de precipitação na Península Antártica. Para o estudo, foram utilizados dados médios mensais de temperatura mínima e máxima do ar e precipitação compreendidos entre os anos de 1969 a 2010 . Os dados foram submetidos ao teste estatístico de Mann-Kendall e ao teste " $t$ " de Student para o coeficiente angular da regressão linear, adotando-se nível de significância para ambos os testes de 5\%. Os resultados indicam aumento de ambas às temperaturas analisadas, com tendência maior para a mínima, principalmente nos meses de outono-inverno. A precipitação demonstrou uma redução para quase todos os meses.
\end{abstract}

Palavras-chave: temperatura máxima, temperatura mínima, teste de Mann-Kendall, mudanças climáticas.

\section{Temperature and Precipitation Trends on the Antarctic Peninsula}

\begin{abstract}
The Antarctic Peninsula has experienced increasing in air temperature which is higher than the global average. Thus, monthly trends in extreme air temperature and precipitation data in the Antarctic Peninsula are evaluate. Monthly averaged minimum and maximum air temperature and precipitation from 1969 to 2010 . Have been analyzed by the Mann-Kendall and Student's t- tests to identify the slope of the linear regression, adopting $5 \%$ of significance level for both tests. The results indicate an increase of both temperatures analyzed, with a larger trend for the minimum air temperature in particular in autumn and winter season. Precipitation showed a decrease trend in almost every months.
\end{abstract}

Keywords: maximum temperature, minimum temperature, Mann-Kendall test, change climate.

\section{Introdução}

A principal consequência do aumento da temperatura para o continente Antártico está ligada ao derretimento das geleiras, seja sobre a superfície e/ou os oceanos. Essa por sua vez irá modificar o albedo da superfície, que influenciará no fluxo de energia térmica e radiativa na superfície. Dentre os fatores inerentes ao derretimento das calotas polares, destacam-se ainda os possíveis desastres ocasionados pelo aumento do nível médio do mar, que pode chegar a 0,98 metros até 2100 , onde de 15 a $35 \%$ deste aumento será em função do derretimento das geleiras (IPCC, 2013).

As observações realizadas no continente Antártico mostraram que as alterações na temperatura do ar não apresentaram uniformidade nas últimas décadas. No entanto, duas regiões do continente demonstraram profundas alterações em decorrência do aumento da temperatura do ar e do mar. A primeira se refere à Ilha Pine e a geleira Thwaites, localizadas no oeste da Antártida. Essa região é extremamente importante para a camada de gelo da Antártida Ocidental, embora tenha apresentado uma descarga de 75 Gt por ano de gelo para o Oceano Austral (Turner e Overland, 2009). Essa descarga mostra que houve um aumento de $77 \%$ desde 1973 , com a metade desse aumento entre 2003 a 2009. No entanto, foi observada uma estabilização 2009 a 2013 por Mouginot et al. (2014).

A outra região em destaque é a Península Antártica que mostrou um aumento de temperatura nos últimos 50 anos. Estudos como os de Broeke e Lipzig (2004) e Turner

Autor de correspondência: Henderson Silva Wanderley, henderson@ufrrj.br. 
et al. (2005) destacaram aumento acentuado na temperatura do ar observado no lado ocidental da Península Antártica. No lado oriental, houve ocorrência de fragmentação na parte norte da plataforma de gelo Larsen, indicando mudanças consideráveis também para essa região.

$\mathrm{O}$ aquecimento na Península Antártica foi resultado da mudança na circulação atmosférica, principalmente o Modo Anular do Hemisfério Sul (SAM, sigla em inglês), da mudança na temperatura da superfície do mar e da desintegração do gelo flutuante sobre os mares de Weddell e Bellingshausen (Turner e Overland, 2009). O aumento de temperatura ocorre juntamente com a desintegração de diversas plataformas de gelo flutuantes.

Desde quando se iniciou as medições na Península, a temperatura média anual da superfície aumentou cerca de 2,7 ${ }^{\circ} \mathrm{C}$, com um aumento na temperatura da ordem de $0,56{ }^{\circ} \mathrm{C}$ por década. No entanto, uma análise detalhada para as temperaturas máxima e mínima são escassas nesta região (Kejna, 2003). Os resultados apresentados por Steig et al. (2009) sugerem que a tendência de aquecimento observada na Península Antártica, desde 1957, se estende ao sul do continente Antártico e ao oeste da Antártida.

$\mathrm{O}$ aumento da temperatura na Península Antártica, resultou na desintegração de uma série de plataformas de gelo flutuantes. Entre essas plataformas inclui-se Larsen-B, que em 2002, liberou $3.250 \mathrm{~km}^{2}$ de gelo, no lado oriental da Península se desintegrando em vários fragmentos. Essa fragmentação liberou 500.000 milhões de toneladas de gelo no oceano Antártico (Turner e Overland, 2009). No entanto, essa fragmentação não proporcionou impactos sobre o nível médio do mar devido a esse gelo já estar flutuando.

O degelo continental é o principal responsável pelo aumento do nível médio do mar. Estudo sobre as geleiras, ao longo da costa da Península, revela que no decorrer dos últimos 50 anos, $87 \%$ dos 244 glaciares estudados demonstraram uma taxa crescente de recuo (Cook et al., 2005). A diminuição da cobertura de gelo na Antártica, seja ela sobre o continente ou oceano, reduz significativamente à quantidade de energia refletida, contribuindo para aumentar a temperatura.

Até o final do século, espera-se uma redução na área de gelo do mar Antártico de aproximadamente 33\% (Bracegirdle et al., 2008), e uma redução de $25 \%$ na extensão de gelo marinho (Arzel et al., 2006). Para o acúmulo de neve do continente Antártico, as previsões sugerem um aumento da precipitação média de 25-50\% (Tuner e Overland, 2009).

A Península Antártica é a região do continente, onde há a maior influência de fenômenos como El Niño-Oscilação Sul (ENOS) (Ivany et al., 2011), oscilações como o SAM e Madden-Julian (Broeke e Lipzig, 2004; Yoo et al., 2012). Esses fenômenos meteorológicos apresentam conexões atmosféricas e oceânicas, as quais juntamente com a cadeia de montanha ao longo da Península causam modi- ficações na distribuição da temperatura, precipitação e vento (Kejna, 2003; Weiss et al., 2011).

Embora existam estudos que busquem compreender como a temperatura e a precipitação estão modificando em escala anual, essa compreensão para as temperaturas extremas e para a precipitação em escala mensal é escassa. Deste modo, surge a necessidade de estudos mais específicos, que visem compreender como essas modificações ocorreram. Diante do exposto, o objetivo deste estudo foi identificar tendências mensais em dados de temperatura extremas e de precipitação na Península Antártica.

\section{Materiais e Métodos}

Para o presente estudo, foram utilizados dados médios mensais de temperatura mínima e máxima do ar e de precipitação provenientes da estação Bellingshausen (62 $12^{\circ}$ S; 58 $56^{\circ} \mathrm{W}$; $14,3 \mathrm{~m}$ de altitude) (http://south.aari.nw.ru/stations/bell/bell_en.html). Esta estação está localizada na Península Fildss, parte acidental da ilha Rei George, Península Antártica. As informações de temperatura e precipitação estão compreendidas entre os anos de 1969 a 2010.

As séries de temperatura e precipitação foram submetidas à análise de regressão linear e ao teste de MannKendall (Mann, 1945; Kendall, 1975), para verificar a existência de possíveis tendências nas sérias temporais mensais. A análise de regressão foi realizada mediante a utilização do teste $\mathrm{t}$ de Student de significância para o coeficiente angular da reta (Gosset, 1939). Esse teste avalia se o coeficiente angular da reta $b$ é significantemente diferente de zero, indicando a presença de tendência na série temporal, considerando a regressão linear de $Y$ com variável aleatória no tempo $X$, Eq. (1).

$$
Y=a+b \mathrm{X}
$$

sendo $Y$ - variável em análise, $X$-tempo, $a$ e $b$ - coeficientes da regressão calculados pelo método dos mínimos quadrados.

A hipótese nula $\left(H_{\mathrm{o}}\right)$ de que não existe alteração, ou seja, $b=0$, é testada por meio do teste t de Student com $n-2$ graus de liberdade, Eq. (2). A hipótese de que não há tendência é rejeitada quando o valor de $t$ calculado é maior, em valor absoluto, do que valor crítico $t_{a / 2, \mathrm{n}-2}$, tabelado, a um determinado nível de significância $a_{0}$, sendo para esse teste adotado nível de significância de 0,05.

$$
t=\frac{r \sqrt{n-2}}{\sqrt{1-r^{2}}}=\frac{b}{s / \sqrt{S S x}}
$$

em que $n$ - tamanho da amostra, $r$ - coeficiente de correlação de Pearson, $s$ - desvio padrão dos resíduos, $b$ - coeficiente angular da reta, $S S_{X}$ - soma dos quadrados da variável independente (tempo em análise de tendência).

O teste de Mann-Kendall é uma análise estatística não-paramétrica o qual é utilizado para identificar tendên- 
cia, que considera a hipótese de estabilidade de uma série temporal, onde a sucessão de valores ocorre de forma independente e a distribuição de probabilidade deve permanecer sempre a mesma (série aleatória simples). Considerando uma série temporal de $Y_{i}$ de $\mathrm{n}$ termos $(1 \leq i \leq n)$, a estatística desse teste é dada pela Eq. (3).

$$
S=\sum_{i=1}^{n-1} \sum_{j=i+1}^{n} \operatorname{sinal}\left(Y_{j}-Y_{i}\right)
$$

em que

$$
\operatorname{sinal}\left(Y_{j}-Y_{i}\right)=\left\{\begin{array}{rcc}
+1 & \text { se } & \left(Y_{j}-Y_{i}\right)>0 \\
0 & \text { se } & \left(Y_{j}-Y_{i}\right)=0 \\
-1 & \text { se } & \left(Y_{j}-Y_{i}\right)<0
\end{array}\right.
$$

Para séries com grande número de termos $(n)$, sob a hipótese nula $\left(H_{\mathrm{o}}\right)$, ausência de tendência na população, $S$ apresenta uma distribuição normal com média zero e variância definida pela Eq. (4). Testando a significância estatística de $S$ para a hipótese alternativa $\left(H_{1}\right)$, usando um teste bilateral a $H_{1}$, esta pode ser rejeitada para grandes valores da estatística $z$, Eq. (5).

$$
\begin{aligned}
& \operatorname{Var}(S)=\frac{\mathrm{n}(\mathrm{n}-1)(2 \mathrm{n}+5)}{18} \\
& z= \begin{cases}\frac{(S-1)}{\sqrt{\operatorname{Var}(S)}} \text { se } S>0 \\
0 \quad \text { se } S=0 \\
\frac{(S+1)}{\sqrt{\operatorname{Var}(S)}} \text { se } S<0\end{cases}
\end{aligned}
$$

Com base na análise da estatística $z$, é feita a decisão final de aceitar ou rejeitar $H_{0}$, ou seja, pode-se confirmar a hipótese de estabilidade dos dados ou rejeitá-la a favor da hipótese alternativa, isto é, a existência de tendência nos dados. O sinal da estatística $z$ indica se a tendência é crescente $(z>0)$ ou decrescente $(z<0)$. Em um teste bilateral para tendência, $H_{1}$ deve ser aceita se $|z| \leq z_{\alpha / 2}$, em que o valor de $z_{\alpha / 2}$ é obtido da tabela na distribuição normal padronizada. Para esse teste também foi adotado nível de significância de 0,05 .

\section{Resultados e Discussão}

Os resultados obtidos com essa análise demonstraram que a temperatura do ar e a precipitação na Península Antártica apresentaram mudanças durante o período de estudo. Para a temperatura do ar foi observado acréscimo tanto na temperatura mínima quanto na máxima, para todos os meses em análise. Embora, o aumento demonstrado pela temperatura mínima seja mais acentuado do que o observado na máxima.

As tendências obtidas para a temperatura mínima, mediante a aplicação do teste de Mann-Kendall, demons- traram valores para $z$ sempre maiores que zero. Esses valores indicam que a tendência apresentada pela temperatura mínima foi positiva, mostrando que a temperatura mínima na Península Antártica aumentou durante o período de análise para todos os meses do ano (Tabela 1). No entanto, apenas os meses de janeiro, fevereiro, maio e agosto evidenciam significância estatística para esse aumento por esse teste.

Resultado análogo também foi encontrado pelo teste de Student, confirmando a tendência de aquecimento encontrada pelo teste de Mann-Kendall (Tabela 1). Porém, além dos meses que mostraram significância decorrente a aplicação do teste de Mann-Kendall, o mês de março também apresentou significância estatística para o aumento da temperatura por esse teste.

O ajuste da linha de tendência apresentado na Fig. 1, mostra que para a maioria dos meses que apresentaram tendência estatisticamente significativa, os maiores valores de temperatura mínima foram obtidos no fim da década de 90 e início dos anos 2000. Esse aumento pode ser uma resposta à redução de gelo sobre os mares de Bellingshausen e Weddell neste período (Weiss et al., 2011).

Janeiro e fevereiro foram os meses de verão que apresentaram tendência estatística significativa para o aumento da temperatura mínima pela aplicação de ambos os testes utilizados no estudo. Para esses meses, o aumento na temperatura mínima foi de 0,21 e $0,22{ }^{\circ} \mathrm{C}$ por década, respectivamente. Nos meses de março e abril o aumento observado na temperatura mínima foi de 0,27 e $0,28^{\circ} \mathrm{C}$ por década (Tabela 1). Esse aumento foi maior do que o apresentado por janeiro e fevereiro, mostrado que o aumento da temperatura nos meses de outono foi maior do que os de verão.

Para os meses de maio a setembro, com exceção a julho, foi observado o maior aumento da temperatura míni-

Tabela 1 - Teste estatístico de Mann-Kendall ( $Z$ ), teste de Student $(t)$ e coeficiente angular da reta $(b / 10$ anos), para a temperatura mínima na estação Bellingshausen.

\begin{tabular}{lccc}
\hline Meses & $Z$ & $t$ & $\beta$ \\
\hline Jan & $2,66^{*}$ & $2,99^{*}$ & 0,21 \\
Fev & $2,65^{*}$ & $2,55^{*}$ & 0,22 \\
Mar & 1,79 & $2,12^{*}$ & 0,27 \\
Abr & 1,01 & 1,31 & 0,28 \\
Mai & $2,77^{*}$ & $2,78^{*}$ & 0,80 \\
Jun & 1,87 & 1,84 & 0,64 \\
Jul & 0,61 & 0,55 & 0,25 \\
Ago & $2,23^{*}$ & $2,66^{*}$ & 0,84 \\
Set & 1,48 & 1,34 & 0,37 \\
Out & 1,03 & 1,01 & 0,19 \\
Nov & 0,99 & 0,64 & 0,08 \\
Dez & 0,31 & 0,12 & 0,01 \\
\hline
\end{tabular}

*significância estatística a 0,05. 

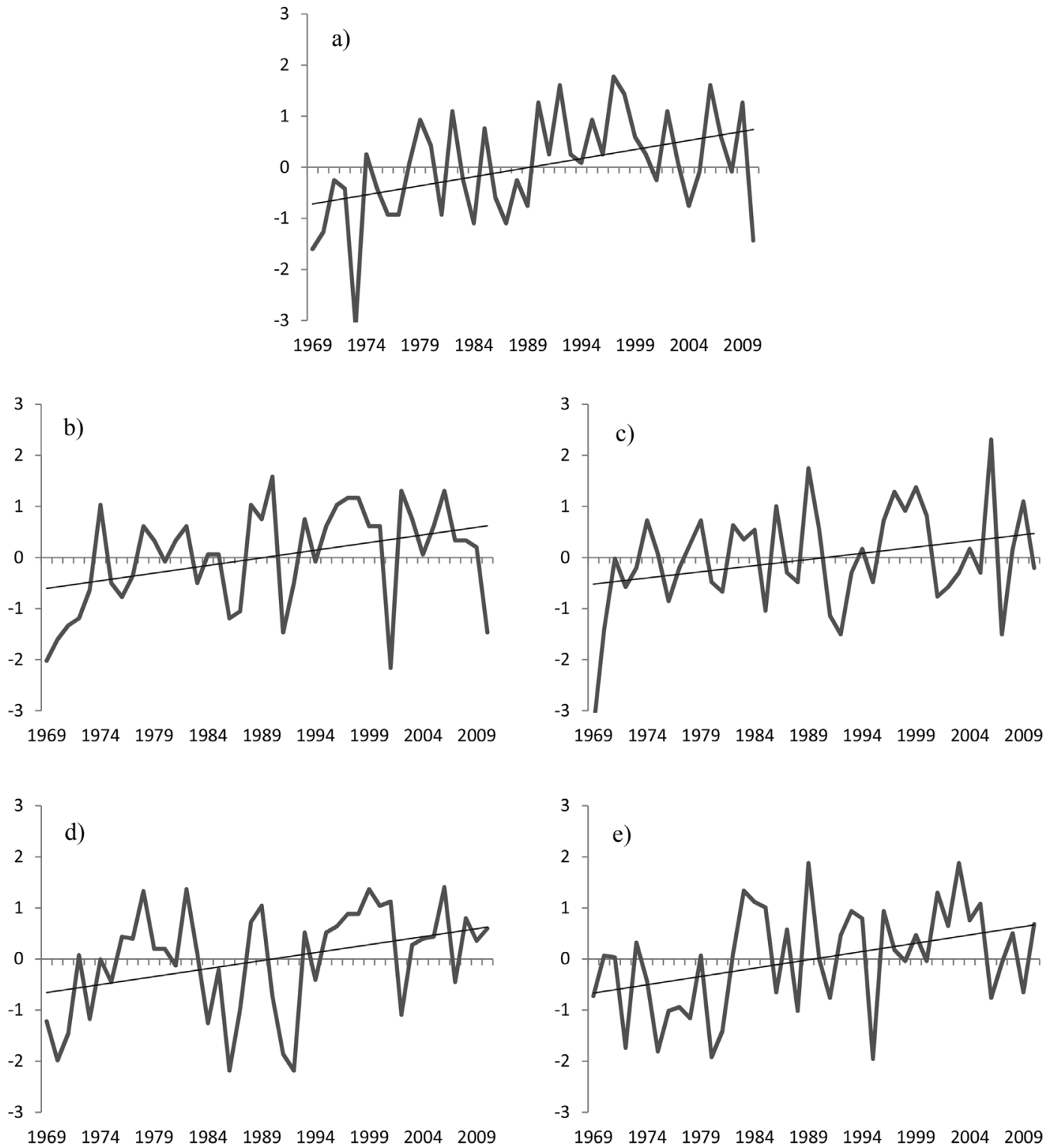

Figura 1 - Regressão linear para os meses que demonstram tendência estatisticamente significativa para a temperatura mínima $\left({ }^{\circ} \mathrm{C}\right)$; a) janeiro, b) fevereiro, c) março, d) maio e e) agosto.

ma. Destes meses, maio e agosto apresentaram significância estatística para o aumento da temperatura mínima por ambos os testes. Esses meses foram os que apresentaram o maior aumento na temperatura mínima. Para maio foi observado aumento de 0,80 e para agosto de $0,84{ }^{\circ} \mathrm{C}$ por década.

Os resultados obtidos neste estudo, assim como os apresentados por Turner et al. (2005), mostram aumento na temperatura dos meses de outono e de inverno superior ao observado para os meses do verão. Parte deste aumento da temperatura durante o inverno se deve ao mecanismo de feedback entre a temperatura do ar e o gelo sobre o oceano. Essa variação na temperatura do ar na Península é influenciada pelo estado do campo térmico sobre as águas do mar de Bellingshausen (King, 1994).
Reduções na cobertura gelo sobre os mares que circundam a Península faz com que o gelo sobre o oceano se fragmente, como o que foi documentada por Bracegirdle et al. (2008). Deste modo, o albedo é modificado e uma quantidade menor de energia é refletida. Sobre a superfície, a redução do gelo tem impacto ainda maior para o aumento da temperatura na Península devido à cor escura do solo. Assim, resultados como os apresentados por Kejna (2003), que encontrou aquecimento cerca de 2,5 vezes maior no inverno do que no verão, podem está apresentando mudanças, uma vez que, os resultados encontrados nesta análise indicam que o aquecimento observado na Península foi até quatro vezes maior no período de inverno do que no verão.

Em observações realizadas por Liu et al. (2004), entre os anos de 1979 a 2002, comprovaram, que a concentração 
de gelo no mar de Bellingshausen e no de Weddell ocidental mostrou uma diminuição de 4 a 10\% por década. Estes resultados ratificam parte das mudanças observadas na temperatura nestas regiões da Antártica. No entanto, a redução na cobertura de gelo não ocorreu de forma uniforme no continente Antártico, pois foi observado aumentado no Mar de Ross, setor do Oceano Austral, principalmente no mês de março (Tuner e Overland, 2009).

A mudança na temperatura observadas na Península Antártica ocorre devido à extensão de gelo marinho entre o mar de Bellingshausen e Weddell causar diferenças climáticas entre as costas leste e oeste da Península. Essas mudanças são oriundas de especificidades da circulação atmosférica e perturbações por fatores orográficos da Península (Kejna, 2003; Weiss et al., 2011).

Essa diminuição no gelo marinho é um dos indicativos para os resultados apresentados neste estudo, com aumento mensal da temperatura mínima superior a $0,80^{\circ} \mathrm{C}$ por década para alguns meses, e de $0,56{ }^{\circ} \mathrm{C}$ por década na temperatura média anual para a região da Península (Tuner e Overland, 2009). As tendências obtidas neste estudo estão de acordo com as observadas por Kejna (2003) e Turner at al. (2005); os quais obtiveram resultados análogos ao desta pesquisa.

A temperatura máxima, semelhante à temperatura mínima, apresentou tendência positiva para todos os meses analisados. Para os meses de janeiro, maio e agosto foi observada tendência estatisticamente significativa, mediante a aplicação do teste de Mann-Kendall. A aplicação do teste $t$ apenas mostrou significância estatística para o mês de maio (Tabela 2). Esse aumento na temperatura também foi observado nas partes oeste e norte da Península Antártica, com tendência estatisticamente significativa de $0,56{ }^{\circ} \mathrm{C}$ por década para a média anual da temperatura, entre os anos de 1951 a 2000 (Tuner e Overland, 2009).

Tabela 2 - Teste estatístico de Mann-Kendall ( $Z$ ), teste de Student $(t)$ e coeficiente angular da reta $(b / 10$ anos), para a temperatura máxima na estação Bellingshausen.

\begin{tabular}{lccc}
\hline Meses & $Z$ & $t$ & $\beta$ \\
\hline Jan & $2,03^{*}$ & 1,86 & 0,17 \\
Fev & 1,44 & 1,21 & 0,10 \\
Mar & 0,85 & 1,19 & 0,12 \\
Abr & 0,57 & 0,71 & 0,10 \\
Mai & $2,49^{*}$ & $2,28^{*}$ & 0,45 \\
Jun & 1,76 & 1,34 & 0,28 \\
Jul & 0,81 & 0,74 & 0,23 \\
Ago & $1,96^{*}$ & 1,76 & 0,42 \\
Set & 0,70 & 0,19 & 0,30 \\
Out & 0,25 & 0,06 & 0,07 \\
Nov & 0,52 & 0,75 & 0,07 \\
Dez & 0,00 & 0,24 & 0,20 \\
\hline
\end{tabular}

*significância estatística a 0,05 .
A temperatura máxima apresentou resultado análogo ao observado na temperatura mínima, com um aumento maior para os meses de outono-inverno. Embora a proporção desse aumento apresentado pela temperatura máxima seja até $50 \%$ menor para alguns meses. Essa comprovação pode ser ratificada para os meses que demonstraram significância estatística, como o mês de agosto, por exemplo (Tabela 2).

Para o mês de agosto, a temperatura máxima apresentou uma tendência de aumento de $0,42{ }^{\circ} \mathrm{C}$ por década, em contrapartida aos $0,84{ }^{\circ} \mathrm{C}$ por década, para a temperatura mínima (Tabela 1). O ajuste linear dos meses que apresentaram tendência estatisticamente significativa para a temperatura máxima foi análogo ao ajuste apresentado pela temperatura máxima, com o maior valor sendo observado entre o fim da década de 90 e início dos anos 2000 (Fig. 2).

Para o mês de janeiro, o aumento na temperatura máxima foi de $0,17{ }^{\circ} \mathrm{C}$ por década, o menor aumento observado na temperatura entre os meses que apresentaram tendência significativa. Para maio e agosto, meses que também apresentaram significância estatística, o aumento na temperatura foi de 0,45 e $0,42{ }^{\circ} \mathrm{C}$ por década, respectivamente. A variabilidade da temperatura máxima dos meses em que foi verificada tendência estatisticamente significativa mostra um aumento acentuado da temperatura também no início da década de 70 ao fim da década de 90 (Fig. 2).

Segundo Ding et al. (2011), o aquecimento observado, principalmente nos meses de inverno, sobre a Antártica ocidental, que se deu iniciou na década de 1970 está associado com aumento da advecção quente para a Antártica. Possivelmente esse aquecimento tenha sido resultado da propagação, em direção aos polos, das ondas de Rossby. Essa propagação fez com que as tendências apresentadas pela temperatura no período do inverno seja maior do que no verão. Isso é decorrente da ação das ondas de Rossby sobre a circulação atmosférica sobre o Mar de Amundsen e de Bellingshausen, causando advecção de ar quente para o continente Antártico que consequentemente, aqueceu o ar na região da Península, como observados nos resultados apresentado nas Figs. 1 e 2.

A advecção quente provocada pela onda de Rossby ocorreu em resposta à mudança na temperatura da superfície do mar na área central do Pacífico tropical. O aquecimento das águas do Pacífico tropical, representado pela fase positiva do ENOS, faz com que a temperatura e a cobertura de gelo na região da Península e áreas adjacentes do oeste da Antártida apresentem impactos significativos. Este padrão é mais evidente na primavera austral, onde a propagação das ondas de Rossby na atmosfera extratropical é mais favorável (Schneider et al., 2012). O resultado dessa modificação foi notório nos meses de primavera, os quais marcaram o início das maiores tendência na temperatura. 
Associada a estas questões, aproximadamente um terço da variabilidade da temperatura anual superficial na Península, entre os anos de 1957 a 2005, foi atribuída à alteração no SAM (Tuner e Overland, 2009). A mudança do SAM resultou em um padrão de aquecimento em toda a Península Antártica e um arrefecimento em grande parte da Antártida oriental. O aquecimento na Península pelo SAM tem sido associado com o fortalecimento dos ventos circumpolar de oeste o qual mudou em resposta a tendência de aumento do índice do SAM desde o início da década de 60 (Marshall, 2003).

$\mathrm{O}$ aumento da temperatura na Península Antártica além de ser influenciada pelas fases dos ENOS e SAM e mudanças da cobertura de gelo, estima-se também que a Oscilação Madden-Julian (OMJ) explica de 10 a 20\% do aquecimento interdecadal observado sobre a Península An-
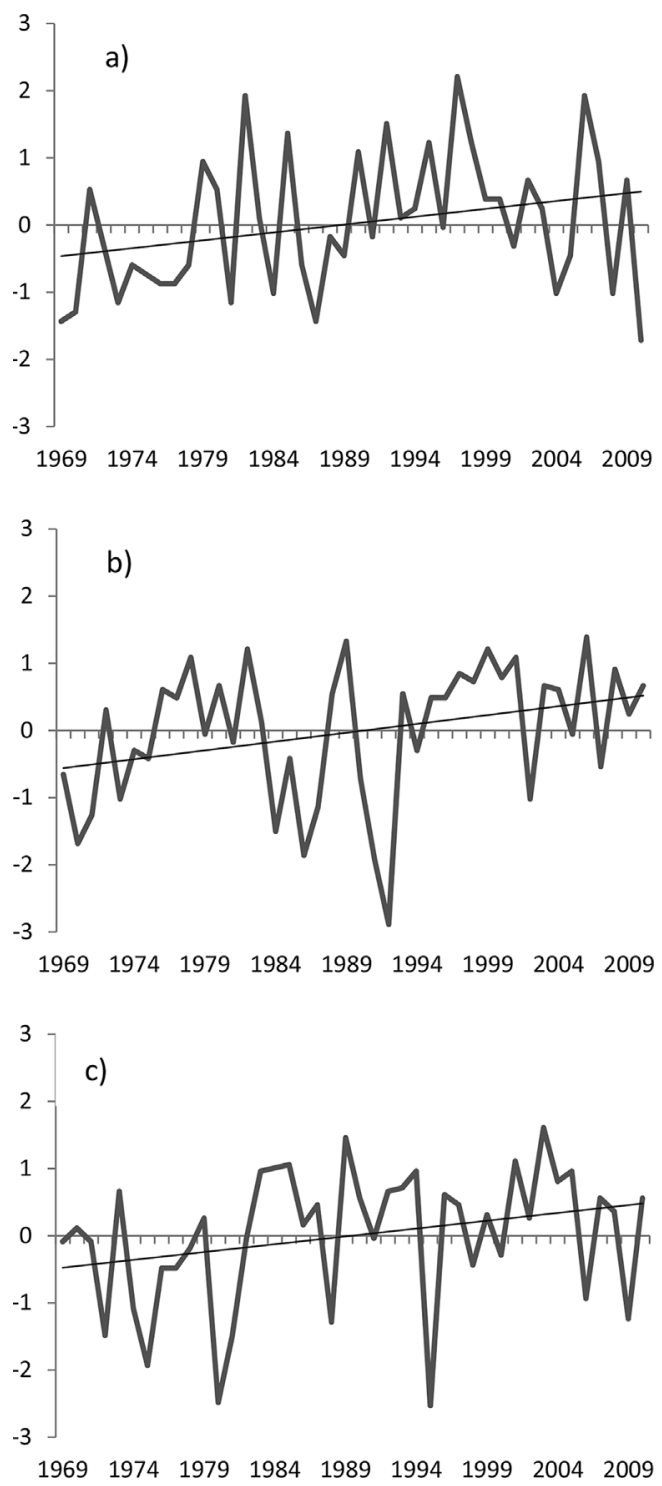

Figura 2 - Regressão linear para os meses que demonstram tendência estatisticamente significativa para a temperatura máxima $\left({ }^{\circ} \mathrm{C}\right)$; a) janeiro, b) maio e c) agosto. tártida (Yoo et al., 2012). Tudo indica que as respostas interdecadais da temperatura sejam em função das mudanças nos padrões espaciais extratropicais para as várias fases da OMJ, em quanto que as mudanças anuais sofram influência maior do ENOS e SAM.

Diferente da temperatura máxima e mínima, a tendência observada na precipitação, para a maioria dos meses foi de decréscimo (Tabela 3). Apenas dois meses dos analisados apresentaram significância estatística. Janeiro para ambos os testes, e agosto para o teste t. Embora a significância estatística não esteja presente para a maioria dos meses, observa-se que apenas nos meses de janeiro, março, julho e outubro apresentou tendência de aumento na precipitação.

Para os meses que demonstraram tendência positiva na análise da precipitação, o aumento não foi superior a $4 \mathrm{~mm}$ por década, para os meses que não apresentaram significância estatística. Para março, julho e outubro o aumento decadal variou de 1,70 a 3,24 $\mathrm{mm}$ por década. Essa modificação observada na precipitação está de acordo com a variabilidade mensal da precipitação na Península. $\mathrm{O}$ maior aumento foi observado para o mês de janeiro, com $8,51 \mathrm{~mm}$ por década, embora janeiro tenha demonstrado significância estatística para esse aumento (Tabela 3).

A regressão linear obtida para o mês de janeiro mostra que a partir da década de 90 a distribuição da precipitação apresentou mudança (Fig. 3). Foram observados os maiores totais pluviométricos nos primeiros anos da década de 90, na região em análise (Fig. 3a). Outra questão relevante observada na Fig. 3a é um aumento na variabilidade da precipitação. Para agosto, mês que também apresentou significância estatística, no entanto para a redução da precipitação, é observada uma redução quase que constante da precipitação, redução esse que foi observada para quase a totalidade dos meses de outono-inverno na Península.

Tabela 3 - Teste estatístico de Mann-Kendall $(Z)$, teste de Student $(t)$ e coeficiente angular da reta $(b / 10$ anos), para a precipitação na estação Bellingshausen.

\begin{tabular}{lccc}
\hline Meses & $Z$ & $t$ & $\beta$ \\
\hline Jan & $2,94^{*}$ & $2,84^{*}$ & 8,51 \\
Fev & $-0,11$ & $-0,18$ & $-0,42$ \\
Mar & 0,70 & 0,60 & 1,70 \\
Abr & $-1,42$ & $-1,01$ & $-2,68$ \\
Mai & $-1,62$ & $-0,96$ & $-2,35$ \\
Jun & $-0,42$ & $-0,67$ & $-2,18$ \\
Jul & 0,35 & 0,80 & 3,24 \\
Ago & $-1,65$ & $-2,08^{*}$ & $-7,42$ \\
Set & $-1,15$ & $-1,02$ & $-2,60$ \\
Out & 0,07 & 0,74 & 2,01 \\
Nov & $-0,68$ & $-0,66$ & $-1,45$ \\
Dez & $-0,48$ & $-0,46$ & $-0,21$ \\
\hline
\end{tabular}

*significância estatística a 0,05 . 

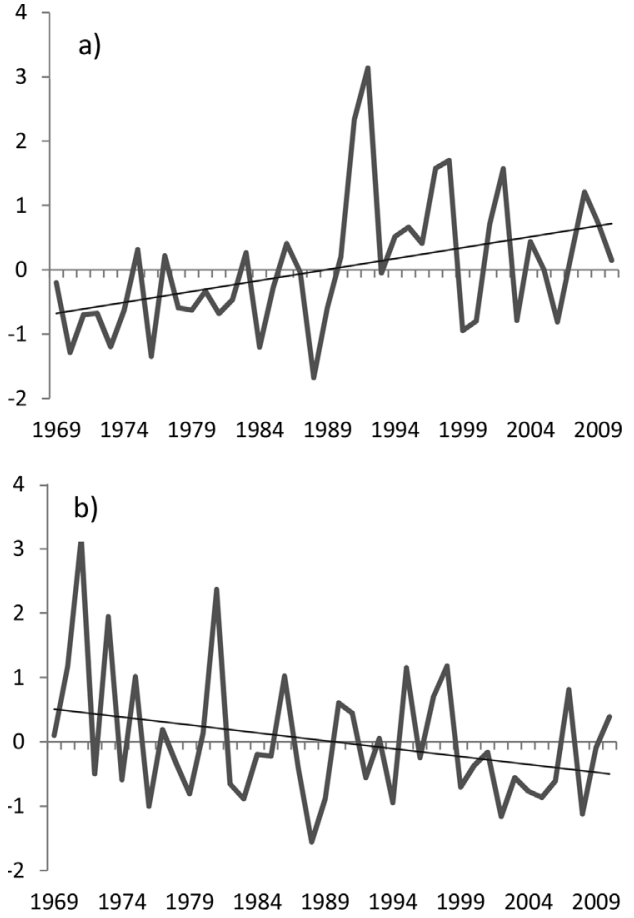

Figura 3 - Regressão linear para os meses que demonstram tendência estatisticamente significativa para a precipitação (mm); a) janeiro e b) agosto.

Os meses que não demonstraram significância estatística para tendência da precipitação apresentaram modificação inferior a $3 \mathrm{~mm}$. Nestes meses, a redução apresentada pela precipitação variou de $-0,21$ a $-2,68 \mathrm{~mm}$ por década. A única exceção foi observada no mês de agosto, com redução decadal na precipitação de $-7,42 \mathrm{~mm}$. No entanto, agosto apresentou significância estatística (Tabela 3).

A maioria dos meses que demonstraram tendência negativa está compreendido no período de outono-inverno. Embora a redução observada da precipitação seja simplória, a análise da precipitação para esse período apresenta peculiar destaque, pois é durante esses meses que ocorre a maior queda de neve na região. A redução da precipitação do outono-inverno pode contribui para uma menor deposição de neve. Essa redução tem impacto direto na redução do albedo, que por sua vez, tende a aumentar a temperatura.

A distribuição da precipitação na Península Antártica é dominada, em escala temporal, pela interação de ciclones frontais existentes sobre o mar de Bellingshausen. Esses ciclones sofrem com a intensificação dos ventos de oeste associados intensidade do SAM. Quando o vórtice circumpolar é intensificando pelo alto índice do SAM, é observado um aumento de até $30 \%$ na precipitação na Península Antártica ocidental, sendo essa mudança estatisticamente significativa (Broeke e Lipzig, 2004).

Quando essas depressões ocorrem no mar de Weddell, o fluxo de sul tende a ser limitado para o lado oriental da Península em função da topografia elevada existente na
Península, a qual se estende por mais de 1,5 km em alguns lugares (Russell et al., 2008). Essa configuração oferece uma divisão eficaz entre as circulações sobre o mar de Weddell e Bellingshausen, fazendo com que a precipitação diminua no período de outono-inverno, como observado na Tabela 3.

Habitualmente, a maioria das depressões sobre o mar Bellingshausen move-se mais lenta para o oeste da Península quando é impulsionada por correntes de ar originárias do quadrante sul (Russell et al., 2008). Assim, a distribuição da precipitação na Península durante os meses de inverno, na maioria dos casos, está associada com a existência de baixa profunda no mar de Bellingshausen, e sua modificação resulta em alteração direta na distribuição da precipitação.

Durante o período de verão, principalmente no mês de janeiro, o qual apresentou aumento estatisticamente significativo para o aumento da precipitação (Tabela 3). A depressões no mar de Bellingshausen é alimentada pelo ar quente originário de latitudes mais baixas. Nestas condições, o gradiente meridional de temperatura na Península é intensificado, reforçada a baroclinia da troposfera, aumentando a atividade frontal, e por consequência as chuvas (Turner et al., 1995).

Para os resultados da distribuição da precipitação obtidos por modelos climáticos, quando executado para a segunda metade do século XX, demonstra-se um pequeno acréscimo na precipitação média em toda a Antártida (Bracegirdle et al., 2008). No entanto, quando essa análise é realizada em escalas de tempo maior, e para regiões específicas, como a Península Antártica, houve indícios de mudança nos resultados, indicando redução da precipitação para essa localidade (Thomas et al., 2008), como apresentado nesta análise.

\section{Conclusões}

A análise realizada para a Península Antártica demonstrou que as temperaturas máxima e mínima analisadas apresentaram tendência positiva, indicando aumento de ambas. A temperatura mínima apresentou um acréscimo maior do que o observado na temperatura máxima. Para a temperatura mínima, todos os meses demonstraram tendência de aumento, no entanto apenas os meses de janeiro, fevereiro, março, maio e agosto foram os que apresentaram tendência estatisticamente significativa, com um maior aquecimento para os meses de outono-inverno, os quais chegaram a apresentar aquecimento decadal superior a $0,80{ }^{\circ} \mathrm{C}$.

A temperatura máxima também demonstrou tendência de aumento para todos os meses do ano, com acréscimo estatisticamente significativo, para os meses de janeiro, maio e agosto. Os maiores acréscimo na temperatura máxima foram observados para os meses de maio e agosto, com um aumento de 0,45 e $0,42{ }^{\circ} \mathrm{C}$, por década, respectivamente. $\mathrm{O}$ aumento na temperatura máxima foi quase a 
metade do observado na temperatura mínima para alguns meses.

A precipitação mostrou tendência negativa para a maioria dos meses, evidenciando diminuição das chuvas sobre a região. No entanto, apenas o mês de agosto demonstrou decréscimo para a precipitação estatisticamente significativa, com uma redução de $7,42 \mathrm{~mm}$ por década. Para o mês de janeiro, foi obtida tendência positiva significativa para o aumento da precipitação de $8,51 \mathrm{~mm}$ por década.

\section{Agradecimento}

Os autores agradecem ao Conselho Nacional de Desenvolvimento Científico e Tecnológico ( $\mathrm{CNPq})$, pelo apoio financeiro a esta pesquisa nos financiamentos dos projetos 232718/2014-8 e 407681/2013-2.

\section{Referências}

ARZEL, O.; FICHEFET, T.; GOOSSE, H. Sea ice evolution over the 20th and 21st centuries as simulated by current AOGCMs. Ocean Modelling, v. 12, p. 401-415, 2006.

BRACEGIRDLE T.J.; CONNOLLEY, W.M.; TURNER, J. Antarctic climate change over the twenty first century. Journal of Geophysical Research, v. 113, p. 13-25, 2008.

BROEKE, M.R.V.D.; LIPZIG, N.P.V. Changes in Antarctic temperature, wind and precipitation in response to the Antarctic Oscillation. Annals of Glaciology, v. 39, p. 119-126, 2004.

COOK, A.J.; FOX, A.J.; VAUGHAN, D.G.; FERRIGNO J.G. Retreating glacier fronts on the Antarctic Peninsula over the past half-century. Science, v. 308, p. 541-544, 2005.

DING, Q.; STEIG, E.J.; BATTISTI, D.S. KÜTTEL, M. Winter warming in west Antarctica caused by central tropical Pacific warming. Nature Geoscience, v. 4, p. 398-403, 2011.

GOSSET, W.S. Student as Statistician. Biometrika, v. 30, p. 210-250, 1939.

IPCC - INTERGOVERNMENTAL PANEL ON CLIMATE CHANGE. Climate Change 2013: The Physical Science Basis. Contribution of Working Group I to the Fifth Assessment Report of the Intergovernmental Panel on Climate Change, Genebra, outubro de 2013.

IVANY, L.C.; BREY, T.; HUBER, M.; BUICK, D.P.; SCHÖNE, B.R. El Niño in the Eocene greenhouse recorded by fossil bivalves and wood from Antarctica. Geophysical Research Letters, v. 38, p. 1-6, 2011.

KEJNA, M. Trends of air temperature of the Antarctic during the period 1958-2000. Polish Polar Research, v. 24, p. 99-126, 2003.

KENDALL, M.G. Rank correlation methods. 4. ed. London: Charles Griffin, p. 196, 1975.
KING, J.C. Recent climate variability in the vicinity of the Antarctic Peninsula. International Journal of Climatology, v. 14, p. 357-369, 1994.

LIU, J.; CURRY, J.A.; MARTINSON, D.G. Interpretation of recent Antarctic sea ice variability. Geophysical Research Letters, v. 31, p. 1-4, 2004.

MANN, H.B. Non-parametric test against trend. Econometrika, v. 13, p. $245-259,1945$.

MARSHALL, G.J. Trends in the Southern Annular Mode from observations and reanalyses. Journal of Climate, v. 16, p. 4134-4143, 2003.

MOUGINOT, J.; RIGNOT, E.; SCHEUCHL, B. Sustained increase in ice discharge from the Amundsen Sea Embayment, West Antarctica, from 1973 to 2013, Geophysical Research Letters., v. 41, p. 1-9. 2014.

RUSSELL, A. MCGREGOR, G.R.; MARSHALL, G.J. Eastern Antarctic Peninsula precipitation delivery mechanisms: process studies and back trajectory evaluation. Atmospheric Science Letters, v. 9, p. 214-221, 2008.

SCHNEIDER, D.; OKUMURA, Y.; DESER, C. Observed Antarctic interannual climate variability and tropical linkages. Journal of Climate, v. 25, p. 4048-4066, 2012.

STEIG, E.J.; SCHNEIDER, D.P.; RUTHERFORD, S.D.; MANN, M.E.; COMISO, J.C.; SHINDELL, D.T. Warming of the Antarctic ice-sheet surface since the 1957 International Geophysical Year. Nature, v. 457, p. 459-462, 2009.

THOMAS, M.; JENKINS, A.; HOLLAND D.; JACOBS, S. Modeling circumpolar deep water intrusions on the Amundsen Sea continental shelf, Antarctica. Geophysical Research Letters, v. 35, p. 1-6, 2008.

TURNER, J.; COLWELL, S.R. ; MARSHALL, G.J.; COPE, T.A.L.; CARLETON, A.M. et al. Antarctic climate change during the last 50 years. International Journal of Climatology, v. 25, p. 279-294, 2005.

TURNER, J.; LACHLAN-COPE, T.A.; THOMAS, J.P.; COLWELL, S.R. The synoptic origins of precipitation over the Antarctic Peninsula. Antarctic Science, v. 7, p. 327337, 1995 .

TURNER, J.; OVERLAND, J. Contrasting climate change in the two polar regions. Polar Research, v. 28, p. 146-164, 2009.

WEISS, A.I.; KING, J.C.; LACHLAN-COPE, T.A.; LADKIN, R.S. Albedo of the ice-covered Weddell and Bellingshausen Sea. The Cryosphere Discuss, v. 5, p. 3259-3289, 2011.

YOO, C.; LEE, S.; FELDSTEIN, S. The impact of the Madden-Julian oscillation trend on the Antarctic warming during the 1979-2008 austral winter. Atmospheric Science Letters, v. 13, p. 194-199, 2012.

All the contents of this journal, except where otherwise noted, is licensed under a Creative Commons Attribution License CC-BY. 